\title{
Special Section Guest Editorial: Computed tomography (CT) at 50 years
}

\author{
Patrick J. La Rivière, ${ }^{\text {a }}$ Rebecca Fahrig, ${ }^{\text {b,c }}$ and Norbert J. Pelc ${ }^{d}$ \\ ${ }^{a}$ University of Chicago, Department of Radiology, Chicago, Illinois, United States \\ ${ }^{b}$ Siemens Healthineers, Innovation, Advanced Therapies, Forchheim, Bavaria, Germany \\ ${ }^{c}$ Friedrich-Alexander Universität, Department of Computer Science 5, Erlangen, Germany \\ ${ }^{\mathrm{d}}$ Stanford University, Department of Radiology, Stanford, California, United States
}

Fifty years ago this month, the first clinical computed tomography image was obtained by Godfrey Hounsfield of EMI and his clinical collaborator James Ambrose at Atkinson Morley hospital in London. While the image is crude by today's technological standards, it clearly visualized a brain tumor and quickly captured the attention of physicians worldwide for the promise it held. It is unquestionably the most significant radiological image since the very first: the famous image of Frau Roentgen's hand acquired 76 years earlier. This special section of the Journal of Medical Imaging marks this anniversary with a collection of five invited review articles as well as nine proffered research papers that represent a cross-section of current investigations in CT.

The five invited historical reviews survey the past 50 years from a variety of technological, computational, and clinical perspectives. In "How CT happened: the early development of medical computed tomography," Schulz and co-authors give a compelling account of the development of the 1971 scanner and the first generations of scanners that followed, recounting stories both familiar and unfamiliar. ${ }^{1}$ Hsieh and Flohr carry the story to the present day in "Computed tomography recent history and future perspectives," ending with a look forward to emerging technologies like photon counting CT. ${ }^{2}$ In "From EMI to AI: a brief history of commercial CT reconstruction algorithms," Crawford and La Rivière survey the computational side of CT over the last fifty years, focusing on four key eras: the algorithmic developments around the 1971 scanner, the optimization and rise to dominance of filtered backprojection (FBP) algorithm, the need for modified FBP to accommodate spiral and cone-beam scanners, and finally the rise of iterative and artificial intelligence (AI) algorithms in recent years. ${ }^{3}$ Fahrig and co-authors consider application-specific CT scanners built around wide, 2D digital detectors mounted on $\mathrm{C}$-arms and other novel cone-beam geometries that have brought $\mathrm{CT}$ technologies into operating and procedure rooms. ${ }^{4}$ Finally, on the clinical side, Dillon guides the reader through key moments in CT neuroimaging. ${ }^{5}$

The nine research papers offer a snapshot of current technical CT research interests and span a wide and representative range of topics. In the realm of emerging hardware and techniques, we see work on system design for a head scanner using a novel x-ray source, ${ }^{6}$ a study of phase contrast CT of the breast, ${ }^{7}$ as well as image reconstruction for photon counting $\mathrm{CT}^{8}{ }^{8}$ In a case of one form of CT helping another, we see microCT used to help characterize the performance of an emerging dedicated breast CT scanner. ${ }^{9}$ Spectral CT is studied for both stand-alone $\mathrm{CT}^{10}$ and as a potential source of improved attenuation correction maps for SPECT. ${ }^{11}$ Finally, image quality and radiation dose are considered from a variety of standpoints, as part of a survey of image quality and dose in 97 different facilities, ${ }^{12}$ as a potential source of noise and metal artifacts to be addressed by novel machine learning techniques, ${ }^{13}$ and finally as a research topic in its own right, requiring novel tools to measure noise texture directly from real images. ${ }^{14}$

The interest in CT, both in the clinical and research settings, has grown steadily in the last 50 years. Nonetheless, CT's decline or demise has been predicted at various points, first with the advent of MRI in the 1980s, then with concerns about radiation dose in the 2000s, but the modality has always rebounded. While MRI certainly proved superior for certain soft-tissue studies, the incredible gains in volumetric speed by CT in the 1990s and 2000s along with its reliable

(C) 2021 Society of Photo-Optical Instrumentation Engineers (SPIE) 
image quality performance and relatively low contraindications have made it the workhorse of the modern radiology department. Meanwhile, concerns about dose have been addressed with improved protocols, system optimization through tube current modulation, and the latest generation of iterative and AI-based reconstruction algorithms. While we cannot predict the future, it seems unwise to bet against CT remaining a source of clinical impact, research innovation, and technical progress.

We expect that readers of this paper may have their own stories and memories they would like to share. Readers interested in doing so can submit their contributions in an email to spiejournals@spie.org with "CT@50" in the subject line. They will be collected and published in this journal in a subsequent editorial.

\section{References}

1. R. A. Schulz et al., "How CT happened: the early development of medical computed tomography," J. Med. Imaging 8(5), 052110 (2021).

2. J. Hsieh and T. Flohr, "Computed tomography recent history and future perspectives," J. Med. Imaging 8(5), 052109 (2021).

3. P. J. La Rivière and C. Crawford, "From EMI to AI: a brief history of commercial CT reconstruction algorithms," J. Med. Imaging 8(5), 052111 (2021).

4. R. Fahrig et al., "Flat-panel conebeam CT in the clinic: history and status," J. Med. Imaging 8(5), 052115 (2021).

5. W. P. Dillon, "50th anniversary of computed tomography: 10 past and future applications in clinical neuroscience," J. Med. Imaging 8(5), 052112 (2021).

6. Y. Luo, "Simulation on system configuration for stationary head CT using linear carbon nanotube x-ray source arrays," J. Med. Imaging 8(5), 052114 (2021).

7. S. Wan et al., "Effect of X-ray energy on the radiological image quality in propagationbased phase-contrast computed tomography of the breast," J. Med. Imaging 8(5), 052108 (2021).

8. M. A. K. Juntunen et al., "Optimizing iterative reconstruction for quantification of calcium hydroxyapatite with photon counting flat-detector computed tomography: a cardiac phantom study," J. Med. Imaging 8(5), 052102 (2021).

9. A. M. Hernandez et al., "High-resolution $\mu \mathrm{CT}$ imaging for characterizing microcalcification detection performance in breast CT," J. Med. Imaging 8(5), 052107 (2021).

10. H. Gong et al., "Deep-learning-based direct synthesis of low-energy virtual monoenergetic images with multi-energy computed tomography," J. Med. Imaging 8(5), 052104 (2021).

11. K. L. Goh and S.-C. Liew, "Dual-energy x-ray approach for object/energy-specific attenuation coefficient correction in single-photon emission computed tomography: effects of contrast agent," J. Med. Imaging 8(5), 052106 (2021).

12. T. B. Smith et al., "Variability in image quality and radiation dose within and across 97 medical facilities," J. Med. Imaging 8(5), 052105 (2021).

13. M. D. Ketcha et al., "Sinogram + image domain neural network approach for metal artefact reduction in low-dose cone-beam CT," J. Med. Imaging 8(5), 052103 (2021).

14. T. B. Smith et al., "Development and validation of an automated methodology to assess perceptual in vivo noise texture in liver CT," J. Med. Imaging 8(5), 052114 (2021).

Patrick J. La Rivière is a professor in the Department of Radiology and the Committee on Medical Physics at the University of Chicago. He received the AB degree in physics from Harvard University and the PhD degree from the Graduate Program in Medical Physics at the University of Chicago. His research interests include tomographic reconstruction in computed tomography, $\mathrm{x}$-ray fluorescence computed tomography, and computational microscopy.

Rebecca Fahrig is head of Innovation for Advanced Therapies at Siemens Healthcare $\mathrm{GmbH}$, and a professor in the Department of Computer Science, at the Pattern Recognition Lab at Friedrich-Alexander Universität, Erlangen, Germany. She received a BSc in physics and MSc in medical biophysics from the University of Toronto, and a $\mathrm{PhD}$ also in medical biophysics from the University of Western Ontario in London, Canada. Her research and pre-development work 
focus on multi-modality image guidance technologies for use during planning, guidance, and assessment of minimally invasive therapies. She is a fellow of the AAPM and AIMBE.

Norbert J. Pelc, professor of radiology, emeritus, at Stanford University, received a BS degree in applied mathematics, engineering, and physics from the University of Wisconsin, and SM and $\mathrm{ScD}$ degrees in medical radiological physics from Harvard University. His research is in technology and the development of applications of diagnostic imaging. He is a member of the National Academy of Engineering and the National Academy of Inventors, and a fellow of SPIE, AAPM, ISMRM, AHA, and AIMBE. 